\title{
Analysis of Outage Performance in Cognitive Radio Networks
}

\author{
Shuqi Liu, Yiming Wang, Yiqi Zhu, Hexin Yang, and Lingjiao Pan
}

\begin{abstract}
This paper evaluates the outage performance of CRNs with mutual interference between SUs and PUs under the underlay approach. We derive the outage probability expression of CRNs, and it is shown that the outage probability of CRNs with considering the interference to $\mathrm{SU}$ from $\mathrm{PU}$ is higher than that of CRNs without considering the interference to $\mathrm{SU}$ from PU. In addition, the outage probability is affected by key network parameters, such as maximum transmit power of SUs, transmit power of $\mathrm{PU}$, interference level of $\mathrm{PU}$, distribution parameter of transmission channel gain or the secondary transmission link (between the secondary transmitter to the secondary receiver) and distribution parameters of interference channel gain or interfering link (from the secondary transmitter to the primary receiver or from the primary transmitter to the secondary receiver). Simulation results have a good agreement with theoretical analysis.
\end{abstract}

Index Terms - Cognitive relay networks, outage probability, Rayleigh fading channel.

\section{INTRODUCTION}

Cognitive radio technology [1] is an efficient means to improve spectrum utilization and has gained much attention in recent years. In cognitive radio networks, secondary users (SUs) are permitted to use the licensed band so long as they protect the data transmission of primary users (PUs) [2]. In the underlay approach, the SU is allowed to use the spectrum of the PUs only when the interference from the SU is less than the interference level which the PU can tolerate. Therefore, to protect the transmission of the PUs in the allocated frequency band, the transmit power of SUs should be constrained. On the other hand, relay communication has been a promising scheme for improving the throughput and coverage of wireless communication systems and has also recently found applications in cognitive radio systems [3]. Inspired by cognitive radio and cooperative relay communication, the authors in [4] proposed the cognitive relay networks (CRNs) which combined cognitive radio technique and cooperative relay technology. Outage probabilities of cognitive relay networks have been presented considering the impact of the

Manuscript received September 20, 2013; revised November 10, 2013 This work was supported by National Natural Science Foundation of China (No.61172056), Doctoral Fund of Ministry of Education of China (20093201110005) from Soochow University and Jiangsu Undergraduate Training Programs for Innovation and Entrepreneurship (No 201311463028 Y)

Shuqi Liu, Yiqi Zhu, Hexin Yang, and Lingjiao Pan are with School of Electrical and Information Engineering, Jiangsu University of Technology, Changzhou, China (e-mail: dxlsq@jsut.edu.cn).

Yiming Wang is with School of Electronics and Information Engineering, Soochow University, Suzhou, China (e-mail: ymwang@ suda.edu.cn). spectrum sensing accuracy in overlay coexistence in [5]. A rough upper bound of outage probability for cognitive relay networks without the maximum transmit power limit was obtained in [6]. In [7], the exact outage probability of an underlay cognitive network using DF (Decoding Forwarding) relaying with best relay selection in Rayleigh fading channels has been studied. The authors in [8] extended the analysis of [7] to Nakagami- $m$ fading channels, an exact outage probability expression was derived, and the impact of various key system parameters was investigated. In [9], the exact outage probability was derived over Rayleigh fading channels in cognitive relay network with the maximum transmit power limit in a spectrum sharing scenario. While these studies only consider the interference to $\mathrm{PU}$ from $\mathrm{SU}$ and ignore the interference to $\mathrm{SU}$ from $\mathrm{PU}$. In practical wireless communication environments, it is not reasonable. No prior work considered mutual interference between PUs and SUs under the underlay approach, which motivates our work.

The paper is organized in five sections. The system model is presented in Section II. The end-to-end outage probability analysis with considering mutual interference between SUs and PUs is given in Section III. Simulation results are given in Section IV to verify the performance of the proposed analysis method, and the conclusions are given in Section V.

\section{SYSTEM MODEL}

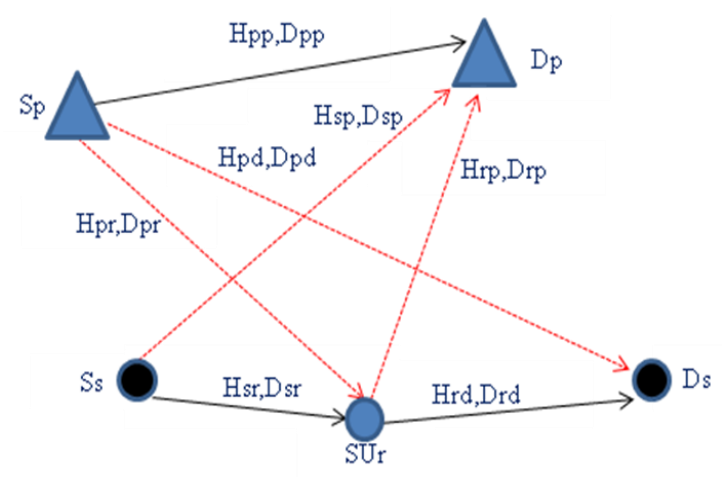

Fig. 1. System model of cognitive relay networks.

We consider an underlay cognitive relay network with mutual interference between $P U s$ and $S U s$, as shown in Fig. 1. In the figure, $S_{p}, D_{p}, S_{s}, S U_{r}$, and $D_{s}$ represent a primary transmitter, a primary receiver, a secondary source, a secondary relay and a secondary destination, respectively. Also we consider a two-hop cognitive relay network in which a source $S_{s}$ transmits data to a destination $D_{s}$ via a relay and there is no direct link between $S_{s}$ and $D_{s}$. The relay mode is regenerative mode, so a relay decodes the received data and 
then forwards it to a secondary destination. $H_{p p}, H_{p r}, H_{p d}, H_{s p}$, $H_{s r}, H_{r p}$, and $H_{r d}$ represent instantaneous channel fading between $S_{p}$ and $D_{p}, S_{p}$ and $S U_{r}, S_{p}$ and $D_{s}, S_{s}$ and $D_{p}, S_{s}$ and $S U_{r}, S U_{r}$ and $D_{p}$, and $S U_{r}$ and $D_{s}$, respectively. $D_{p p}, D_{p r}, D_{p d}$, $D_{s p}, D_{s r}, D_{r p}$, and $D_{r d}$ represent the link distance between $S_{p}$ and $D_{p}, S_{p}$ and $S U_{r}, S_{p}$ and $D_{s}, S_{s}$ and $D_{p}, S_{s}$ and $S U_{r}, S U_{r}$ and $D_{p}$, and $S U_{r}$ and $D_{s}$, respectively.

The channel impulse response is assumed to relate with path loss and an independent fading effect as $H_{m n}=X_{m n}\left(D_{m n}\right)^{-\frac{\alpha}{2}},\{m, n \in(p, s, r, d)\}$ where $X_{m n}$ and $\alpha$ denote the fading coefficient and the pathloss exponent, respectively. The fading coefficient, $X_{m n}$, is a complex Gaussian random variable with mean zreo and variance $\sigma_{m n}{ }^{2}$. Hence, the instantaneous channel gain $\left|H_{m n}\right|^{2}=\left|X_{m n}\right|^{2}\left(D_{m n}\right)^{-\alpha}$ is an exponential distributed random variable with distribution parameter $\lambda_{m n}$. It is assumed here that all channels are slow fading channels and all channel state information can be obtained by RTS/CTS of IEEE802.11.

In the underlay approach of this paper, the transmission of the secondary user is allowed as long as it does not generate harmful interference at primary destination $D_{p}$, and this is achieved by imposing the following transmit power constraints at secondary source $\mathrm{S}_{\mathrm{s}}$ and relay $S U_{r}$.

$$
P_{s} \leq \min \left\{\frac{I_{t h}}{\left|H_{s p}\right|^{2}}, P_{\max }\right\}, \quad P_{r} \leq \min \left\{\frac{I_{t h}}{\left|H_{r p}\right|^{2}}, P_{\max }\right\}
$$

where $I_{t h}$ is the interference temperature constraint, and $P_{\max }$ is the maximum transmit power available at $S_{s}$ and $S U_{r}$. We consider a cognitive network in which the transmission from $S U$ source to $S U$ destination takes place in two hops. During the first hop, $S_{s}$ transmits to $S U_{r}$ with an average power of $P_{s}$, and $S U_{r}$ fully decodes the message based on the received signal. Then, $S U_{r}$ transmits a re-encoded message with an average power of $P_{r}$ to $D_{s}$ during the second hop. Therefore, the signal-to-interference and noise ratio (SINR) of the first hop and the SINR of the second hop can be obtained respectively by

$$
\gamma_{1 r}=\frac{P_{s} \cdot\left|H_{s r}\right|^{2}}{P_{p} \cdot\left|H_{p r}\right|^{2}+N_{0}}, \quad \gamma_{2 r}=\frac{P_{r} \cdot\left|H_{r d}\right|^{2}}{P_{p} \cdot\left|H_{p d}\right|^{2}+N_{0}}
$$

where $P_{p}$ is the transmit power of primary transmitter, and $\mathrm{N}_{0}$ is noise power. As regards a $D F$ protocol, the end-to-end output SINR at destination $D_{s}$ can be tightly approximated in the high SINR regime as follows [10]:

$$
\gamma_{r}=\min \left\{\gamma_{1 r}, \gamma_{2 r}\right\}
$$

\section{OUTAGE PERFORMANCE ANALYSIS}

In this section, we investigate the outage performance of the previously described cognitive relay networks and analyze the end-to-end outage probability. The $S U_{i}$ operates in half-duplex mode. The end-to-end mutual information of $S_{s^{-}}>S U_{r^{-}}>D_{s}$ is given by

$$
I_{r}=\frac{1}{2} \log _{2}\left(1+\gamma_{r}\right)
$$

The outage probability of the system is defined as the probability that the instantaneous mutual information falls below a predefined rate threshold $C_{t h}$. Therefore, the outage probability can be expressed as

$$
\begin{aligned}
P_{\text {out }} & =\operatorname{Pr}\left\{I_{r}<C_{\text {th }}\right\}=\operatorname{Pr}\left\{\gamma_{r}<2^{2 . C_{\text {th }}}-1\right\} \\
& =F_{r}\left(2^{2 . C_{\text {th }}}-1\right)
\end{aligned}
$$

Next, we discuss the cumulative distribution function (CDF) of $\gamma_{1 r}$ and $\gamma_{2 r}$, respectively. For the first hop, the CDF of $\gamma_{1 r}$ in (2) can be given by

$$
\begin{aligned}
& F_{1 r}(\gamma)=\operatorname{Pr}\left\{\gamma_{1 r} \leq \gamma\right\}=\operatorname{Pr}\left\{\frac{P_{s} \cdot\left|H_{s r}\right|^{2}}{P_{p} \cdot\left|H_{p r}\right|^{2}+N_{0}} \leq \gamma\right\} \\
& =\underbrace{\operatorname{Pr}\left\{\frac{\left|H_{s p}\right|^{2}}{P_{p} \cdot\left|H_{p r}\right|^{2}+\left.N_{0}\right|^{2}} \leq \gamma ; \frac{I_{t h}}{\left|H_{s p}\right|^{2}} \leq P_{\max }\right\}}_{A} \\
& +\underbrace{\operatorname{Pr}\left\{\frac{P_{\max } \cdot\left|H_{s r}\right|^{2}}{P_{p} \cdot\left|H_{p r}\right|^{2}+N_{0}} \leq \gamma ; \frac{I_{t h}}{\left|H_{s p}\right|^{2}}>P_{\max }\right\}}_{B}
\end{aligned}
$$

For analysis convenience, we define random variable $V=\frac{I_{t h}}{\left|H_{s p}\right|^{2}} \cdot\left|H_{s r}\right|^{2}$, the CDF of $V$ is given by

$$
\begin{aligned}
F_{V}(v) & =\operatorname{Pr}\{V \leq v\} \\
& =\operatorname{Pr}\left\{\frac{I_{t h}}{\left|H_{s p}\right|^{2}} \cdot\left|H_{s r}\right|^{2} \leq v,\left|H_{s p}\right|^{2} \geq \frac{I_{t h}}{P_{\max }}\right\} \\
& =e^{-\lambda_{s p} \cdot \frac{I_{t h}}{P_{\max }}}-\frac{\lambda_{s p} \cdot I_{t h} \cdot e^{-\lambda_{s p} \cdot \frac{I_{t h}}{P_{\max }}}}{\lambda_{s p} \cdot I_{t h}+\lambda_{s r} \cdot v} \cdot e^{-\frac{\lambda_{s r}}{P_{\max }} v}
\end{aligned}
$$

where $f_{V}(v)=\frac{d F_{V}(v)}{d v}$ is probability density function (PDF).

$$
\begin{aligned}
A & =\operatorname{Pr}\left\{\frac{V}{P_{p} \cdot\left|H_{p r}\right|^{2}+N_{0}} \leq \gamma\right\} \\
& =\operatorname{Pr}\left\{V \leq\left(P_{p} \cdot\left|H_{p r}\right|^{2} \cdot \gamma+N_{0} \cdot \gamma\right)\right\} \\
& ==e^{-\lambda_{s p} \cdot \frac{I_{l h}}{P_{\max }}}-\frac{\lambda_{p r} \cdot \lambda_{s p} I_{t h}}{\lambda_{s r} \cdot P_{p} \cdot \gamma} \cdot e^{\frac{\left(\lambda_{s p} I_{t h}+\lambda_{s r} \cdot N_{0} \cdot \gamma\right) \cdot \lambda_{p r}}{\lambda_{s r} \cdot P_{p} \cdot \gamma}} \cdot \Gamma(0, \Delta)
\end{aligned}
$$

where $\Gamma(0, x)=\int_{x}^{\infty} \frac{e^{-t}}{t} d t$ denotes the incomplete Gamma function,

$$
\Delta=\frac{\lambda_{s p} \cdot I_{t h}}{P_{\max }}+\frac{\lambda_{p r} \cdot N_{0}}{P_{p}}+\frac{\lambda_{s r} \cdot N_{0} \cdot \gamma}{P_{\max }}+\frac{\lambda_{p r} \cdot \lambda_{s p} I_{t h}}{\lambda_{s r} \cdot P_{p} \cdot \gamma} .
$$


The term $B$ is as follows

$$
\begin{aligned}
B & =\operatorname{Pr}\left\{\frac{P_{\max } \cdot\left|H_{s r}\right|^{2}}{P_{p} \cdot\left|H_{p r}\right|^{2}+N_{0}} \leq \gamma ;\left|H_{s p}\right|^{2}<\frac{I_{t h}}{P_{\max }}\right\} \\
& =1-e^{-\frac{\lambda_{s p} \cdot I_{t h}}{P_{\max }}}-\frac{P_{\max .} \lambda_{p r}}{P_{\max .} \lambda_{p r}+\lambda_{s r} \cdot P_{p} \cdot \gamma} e^{-\frac{\lambda_{s r} \cdot N_{0} \cdot \gamma}{P_{\max }}} \\
& +\frac{P_{\max .} \lambda_{p r}}{P_{\text {max }} \cdot \lambda_{p r}+\lambda_{s r} \cdot P_{p} \cdot \gamma} e^{-\left(\frac{\lambda_{s r} \cdot N_{0}, \gamma+\lambda_{s p} \cdot I_{t h}}{P_{\max }}\right)}
\end{aligned}
$$

where $\lambda_{s p}, \lambda_{s r}$, and $\lambda_{p r}$ represent distribution parameters of exponential distributed random variables $\left|H_{s p}\right|^{2},\left|H_{s r}\right|^{2}$, $\left|H_{p r}\right|^{2}$, respectively.

For the second hop, the CDF of $\gamma_{2 r}$ in (2) is given by

$$
\begin{aligned}
& F_{2 r}(\gamma)=\operatorname{Pr}\left\{\gamma_{2 r} \leq \gamma\right\}=\operatorname{Pr}\left\{\frac{P_{r} \cdot\left|H_{r d}\right|^{2}}{P_{p} \cdot\left|H_{p d}\right|^{2}+N_{0}} \leq \gamma\right\} \\
& =\underbrace{\operatorname{Pr}\left\{\frac{\frac{I_{t h}}{\left|H_{r p}\right|^{2}} \cdot\left|H_{r d}\right|^{2}}{P_{p} \cdot\left|H_{p d}\right|^{2}+N_{0}} \leq \gamma ; \frac{I_{t h}}{\left|H_{r p}\right|^{2}} \leq P_{\text {max }}\right\}}_{C} \\
& +\underbrace{\operatorname{Pr}\left\{\frac{P_{\max } \cdot\left|H_{r d}\right|^{2}}{P_{p} \cdot\left|H_{p d}\right|^{2}+N_{0}} ; \frac{I_{t h}}{\left|H_{r p}\right|^{2}}>P_{\max }\right\}}_{D}
\end{aligned}
$$

Using similar analysis method with $\gamma_{1 r}$, the terms C and D are given by

$$
\begin{aligned}
C & =\operatorname{Pr}\left\{\frac{\left|H_{r p}\right|^{2} \cdot\left|H_{r d}\right|^{2}}{P_{p} \cdot\left|H_{p d}\right|^{2}+N_{0}} \leq \gamma ; \frac{I_{t h}}{\left|H_{r p}\right|^{2}} \leq P_{\max }\right\} \\
& =e^{-\lambda_{r p} \cdot \frac{I_{t h}}{P_{\max }}}-\frac{\lambda_{p d} \cdot \lambda_{r p} I_{t h}}{\lambda_{r d} \cdot P_{p} \cdot \gamma} \cdot e^{\frac{\left(\lambda_{r p}, I_{t h}+\lambda_{r d} \cdot N_{0} \cdot \gamma\right) \cdot \lambda_{p d}}{\lambda_{r d} \cdot P_{p} \cdot \gamma}} \cdot \Gamma(0, \nabla)
\end{aligned}
$$

where $\nabla=\frac{\lambda_{r p} \cdot I_{t h}}{P_{\max }}+\frac{\lambda_{p d} \cdot N_{0}}{P_{p}}+\frac{\lambda_{r d} \cdot N_{0} \cdot \gamma}{P_{\max }}+\frac{\lambda_{p d} \cdot \lambda_{r p} I_{t h}}{\lambda_{r d} \cdot P_{p} \cdot \gamma}$.

$$
\begin{aligned}
D & =\operatorname{Pr}\left\{\frac{P_{\max } \cdot\left|H_{r d}\right|^{2}}{P_{p} \cdot\left|H_{p d}\right|^{2}+N_{0}} ; \frac{I_{t h}}{\left|H_{r p}\right|^{2}}>P_{\max }\right\} \\
& =1-e^{-\frac{\lambda_{r p} \cdot I_{t h}}{P_{\max }}}-\frac{P_{\max } \cdot \lambda_{p d}}{P_{\max .} \lambda_{p d}+\lambda_{r d} \cdot P_{p} \cdot \gamma} e^{-\frac{\lambda_{r d} \cdot N_{0}, \gamma}{P_{\max }}} \\
& +\frac{P_{\max .} \lambda_{p d}}{P_{\max .} \lambda_{p d}+\lambda_{r d} \cdot P_{p} \cdot \gamma} e^{-\left(\frac{\lambda_{r d} \cdot N_{0} \cdot \gamma+\lambda_{r p} \cdot I_{t h}}{P_{\max }}\right)}
\end{aligned}
$$

where $\lambda_{r p}, \lambda_{r d}$, and $\lambda_{p d}$ represent distribution parameters of exponential distributed random variables $\left|H_{r p}\right|^{2},\left|H_{r d}\right|^{2}$, $\left|H_{p d}\right|^{2}$, respectively. Then the CDF of $\gamma_{r}$ can be represented as

$$
\begin{aligned}
F_{r}(\gamma) & =\operatorname{Pr}\left\{\min \left\{\gamma_{1 r}, \gamma_{2 r}\right\} \leq \gamma\right\} \\
& =1-\operatorname{Pr}\left\{\min \left\{\gamma_{1 r}, \gamma_{2 r}\right\}>\gamma\right\} \\
& =1-\left[1-F_{1 r}(\gamma)\right] \cdot\left[1-F_{2 r}(\gamma)\right] \\
& =F_{1 r}(\gamma)+F_{2 r}(\gamma)-F_{1 r}(\gamma) \cdot F_{2 r}(\gamma)
\end{aligned}
$$

\section{SimUlations AND ANALYSIS}

In this section, we examine the performance of cognitive relay networks based on the outage probability. Simulations are conducted to verify the outage probabilities derived from (5), and the results closely match the analysis, as shown in Figs. 2-4. All the theoretical and simulation results are derived in an independent but not identically distributed (INID) Rayleigh fading environment. It is assumed that noise power $\mathrm{N}_{0}$ is equal to 1 . And $\lambda_{p r}, \lambda_{p d}, \lambda_{s p}, \lambda_{r p}, \lambda_{s r}$, and $\lambda_{r d}$ represent distribution parameters of exponential distributed random variables $\left|H_{p r}\right|^{2},\left|H_{p d}\right|^{2},\left|H_{s p}\right|^{2},\left|H_{r p}\right|^{2},\left|H_{s r}\right|^{2}$, and $\left|H_{r d}\right|^{2}$, respectively.

Fig. 2 gives the curves of outage probability versus the maximum transmission power of SUs with different secondary transmission channel gain distribution parameters $\lambda_{s r}$ and $\lambda_{r d}$. We have set $\lambda_{p r}=\lambda_{p d}=10, \lambda_{s p}=\lambda_{r p}=10$, $P p=10 \mathrm{~dB}, I_{t h}=5 \mathrm{~dB}$ and $C_{t h}=0.5 \mathrm{bps} / \mathrm{Hz}$. From the figure, we can see that the exact analytic results are matched with the simulated ones considering the mutual interference between PUs and SUs or without considering the interference to SU from PU for $\lambda_{s r}=\lambda_{r d}=5$ and $\lambda_{s r}=\lambda_{r d}=2$, respectively. The outage performance of CRNs considering the mutual interference between PUs and SUs is worse than that of CRNs without considering the interference to SU from PU with the same channel parameters and maximum transmission power $P_{\max }$. The outage probability decreases with increasing of the maximum transmission power $P_{\max }$. When the maximum transmission power $P_{\max }$ is fixed, the larger transmission channel parameters, $\lambda_{s r}$ and $\lambda_{r d}$, are, the higher the outage probability is Fig. 2 also illustrates the outage performance heavily relies on the channel quality of the secondary transmission links. And $\lambda_{s r}$ and $\lambda_{r d}$ determine the channel quality of the secondary transmission links.

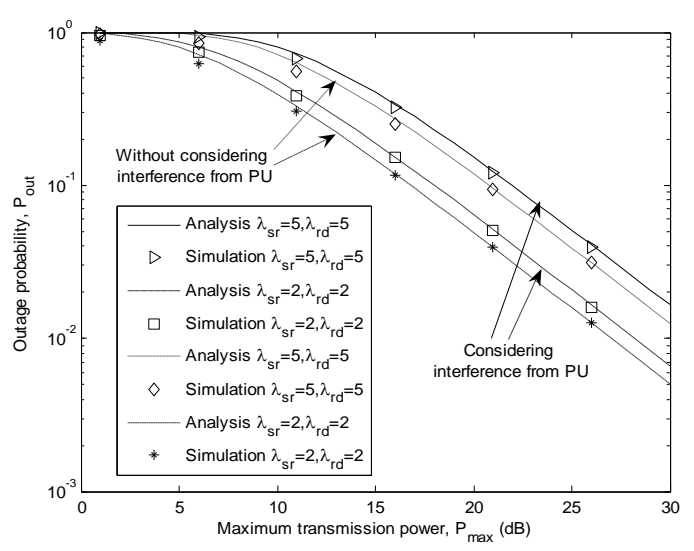

Fig. 2. Outage probabilities, $P_{\text {out }}$ versus maximum transmission power, $P_{\max }$ under different $\lambda_{s r}$ and $\lambda_{r d}\left(P p=10 \mathrm{~dB}, \lambda_{p r}=\lambda_{p d}=10, \lambda_{s p}=\lambda_{r p}=10\right.$, $\left.I_{t h}=5 \mathrm{~dB}, C_{t h}=0.5 \mathrm{bps} / \mathrm{Hz}\right)$. 
In Fig. 3, the curves of outage probability versus interference threshold are plotted using the following parameters: $\lambda_{p r}=\lambda_{p d}=2, \quad \lambda_{s p}=\lambda_{r p}=1, \quad \mathrm{Pp}=10 \mathrm{~dB}$, $P_{\text {max }}=20 \mathrm{~dB}$, and $C_{t h}=0.5 \mathrm{bps} / \mathrm{Hz}$. From Fig. 3 , we can see the outage probability of CRNs with considering the interference to SU from PU or not considering the interference to SU from PU decreases with increase of interference threshold for $\lambda_{s r}=\lambda_{r d}=3$ and $\lambda_{s r}=\lambda_{r d}=5$, respectively. For given $I_{t h}$, the outage performance of system degrades with increase of $\lambda_{s r}$ and $\lambda_{r d}$. When $I_{t h} \rightarrow 0$, the outage probability is close to one, and it effectively means that $D_{p}$ cannot tolerate any additional interference, permitting no secondary transmission. Similarly when $I_{t h} \rightarrow \infty$, the outage probability is very small, it effectively means that $D_{p}$ can tolerate any additional interference and secondary transmission is always feasible.

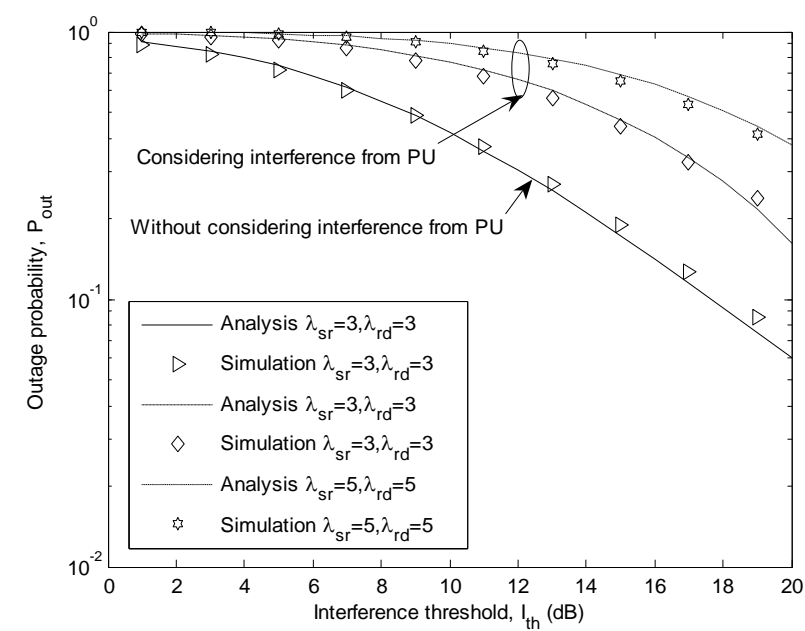

Fig. 3. Outage probabilities, $P_{\text {out }}$ versus interference threshold, $I_{t h}$ under different $\lambda_{s r}$ and $\lambda_{r d}\left(P_{p}=10 \mathrm{~dB}, \lambda_{p r}=\lambda_{p d}=2, \lambda_{s p}=\lambda_{r p}=1, P_{\max }=20 \mathrm{~dB}\right.$, $\left.C_{t h}=0.5 \mathrm{bps} / \mathrm{Hz}\right)$.

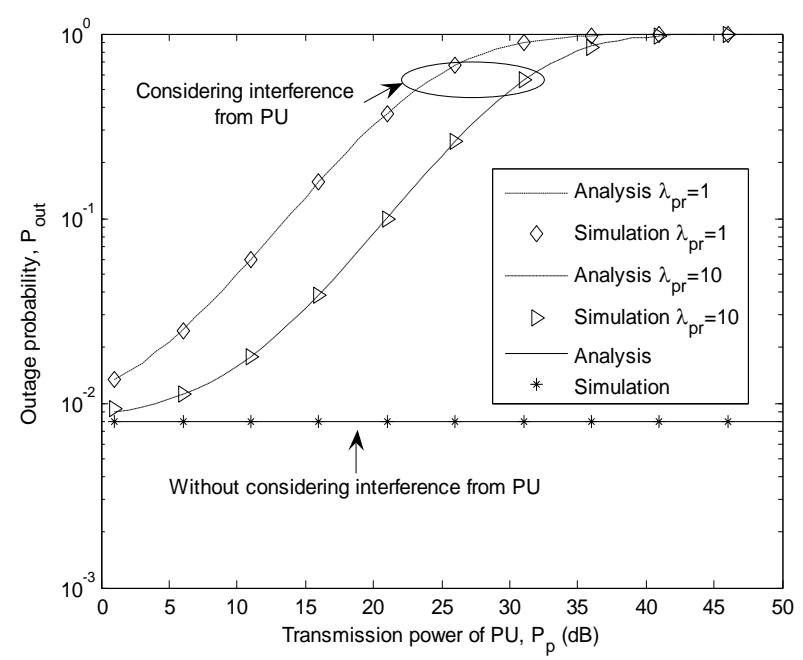

Fig. 4. Outage probabilities, $P_{\text {out }}$ versus transmission power of PU, $P_{p}$ under different $\lambda_{p r}\left(P_{\max }=20 \mathrm{~dB}, \lambda_{s p}=\lambda_{r p}=\lambda_{p d}=10, \lambda_{s r}=\lambda_{r d}=1, I_{t h}=5 \mathrm{~dB}\right.$,

$$
\left.C_{t h}=0.5 \mathrm{bps} / \mathrm{Hz}\right) \text {. }
$$

Fig. 4 depicts the relationship between the outage probability of system and the transmission power of $P U$ for $\lambda_{p r}=1$ and $\lambda_{p r}=10$, respectively, with $P_{\max }=20 \mathrm{~dB}, I_{t h}=$ $5 \mathrm{~dB}, C_{t h}=0.5 \mathrm{bps} / \mathrm{Hz}, \lambda_{s r}=\lambda_{r d}=1$, and $\lambda_{s p}=\lambda_{r p}=\lambda_{p d}=10$.
The outage probability of the system is a constant and it is not affected by the transmission power of PU, when the interference to SU from PU is not considered. The outage probability of the system becomes larger when the interference to SU from PU is considered, and it increases with the increase of $P_{p}$. Notice that increasing $P_{p}$ implies increasing interference to SU from PU. When $P_{p}$ is much smaller than $P_{\max }$, the outage probability is quite close to the outage probability without considering the interference to SU from PU. The outage probability increase due to larger interference becomes much more pronounced when $P_{p}$ is larger than $P_{\max }$. Hence, the interference to $\mathrm{SU}$ from $\mathrm{PU}$ should not be ignored when we analyze the outage performance of CRNs. For fixed $P_{p}$, the outage performance of the system improves with increase of $\lambda_{p r}$, which also illustrates the outage performance of the system depending on interfering link. At the same time, we also observe that the impact of $\lambda_{p d}$ on the outage probability of the system is similar to that of $\lambda_{p r}$.

\section{CONCLUSIONS}

In this paper, the exact outage probability expression of cognitive relay network considering mutual interference between PUs and SUs is derived in Rayleigh fading channels, which provides an efficient means to investigate the impact of network parameters on the outage performance of CRNs. The theoretical analysis is validated by simulation results. Both theoretical analysis and simulation reveal that both the interference to SU from PU and the interference to PU from SU can not be ignored and they have an important impact on outage performance of CRNs. Our results are very important to research routing of cognitive relay networks based on the outage probability.

\section{REFERENCES}

[1] S. Haykin, "Cognitive radio: brain-empowered wireless communications," IEEE J. Sel. Areas Commun., vol. 23, no. 2, pp. 201-220, Feb. 2005.

[2] A. Goldsmith, S. A. Jafar, I. Maric, and S. Srinivasa, "Breaking spectrum gridlock with cognitive radios: an information theoretic perspective," Proceedings of the IEEE, vol. 97, no. 5, pp. 894-914, May 2009.

[3] M. O. Hasna and M. S. Alouini, "End-to-end performance of transmission systems with relays over Rayleigh-fading channels," IEEE Trans. Wireless Commun., vol. 2, no. 6, pp. 1126-1131, Nov. 2003.

[4] Q. Zhang, J. Jia, and J. Zhang, "Cooperative relay to improve diversity in cognitive radio networks," IEEE Commun. Mag., vol. 47, no. 2, pp. 111-117, Feb. 2009

[5] K. Lee and A. Yener, "Outage performance of cognitive wireless relay networks," in Proc. IEEE Global Commun. Conf., pp. 1-5, Nov. 2006.

[6] Y. Guo, G. Kang, N. Zhang, W. Zhou, and P. Zhang, "Outage performance of relay-assisted cognitive-radio system under spectrum-sharing constraints," IET Electron. Lett., vol. 46, no. 2, pp. 182-184, Jan. 2010.

[7] J. Lee, H. Wang, J. G. Andrews, and D. Hong, "Outage probability of cognitive relay networks with interference constraints," IEEE Trans. Wireless Commun., vol. 10, no. 2, pp. 390-395, Feb. 2011.

[8] C. Zhong, T. Ratnarajah, and K. Wong, "Outage analysis of decode-and-forward cognitive dual-hop systems with the interference constraint in Nakagami-m fading channels," IEEE Trans. Veh. Technol., vol. 60, no. 6, pp. 2875-2879, July 2011. 
[9] Z. Yan, X. Zhang, and W. Wang, "Exact outage performance of cognitive relay networks with maximum transmit power limits," IEEE Commun. Lett., vol. 15, no. 12, pp. 1317-1319, Dec. 2011.

[10] A. Bletsas, A. Khisti, D. P. Reed, and A. Lippman, "A simple cooperative diversity method based on network path selection," IEEE J. Sel. Areas Commun., vol. 24, no. 3, pp. 659-672, Mar. 2006.

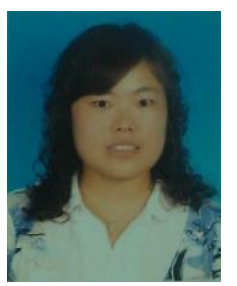

Shuqi Liu received her B.S. Degree in School of Information Engineering from Zhengzhou University, Zhengzhou, in 2002, and M.S. degree in Electronics and Information Engineering from Soochow University, Suzhou, China, in 2005. Since 2005, she has been a lecturer at School of Electrical and Information Engineering, Jiangsu University of Technology, Changzhou, China. She is currently pursuing her Ph.D. in signal and information processing at Soochow University. Her research interests include adaptive signal processing, connectivity analysis and routing design for cognitive radio networks.

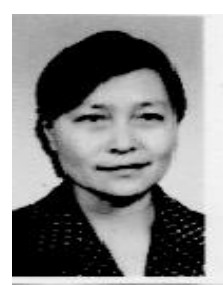

Yiming Wang received her B.S. Degree in electronic device and Ph.D. degree in communications engineering from Nanjing University of Posts and Telecommunications, Nanjing, China, in 1982 and 2006 respectively. She is now a full Professor and $\mathrm{Ph} . \mathrm{D}$. supervisor at the school of Electrical and Information Engineering, Soochow University, China, where she has been leading research activities in the area of cognitive radio, multimedia communication and wireless communications. Her current research interests include communication signal processing, broadband wireless communications and cognitive radio.

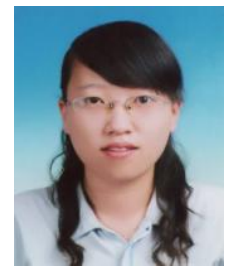

Yiqi Zhu received her B.S. Degree in School of Telecommunication and Information Engineering from Nanjing University of Posts and Telecommunications, Nanjing, in 2008, and M.S. degree in School of Telecommunication and Information Engineering from Nanjing University of Posts and Telecommunications, Nanjing, in 2011. Since 2011, she has been an assistant at School of Electrical and Information Engineering of Jiangsu University of Technology, Changzhou, China. Her research interest includes cognitive radio networks.

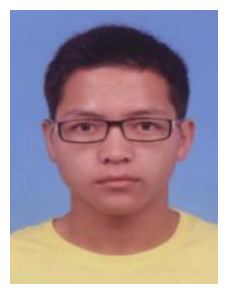

Hexin Yang was born in October 1990. Now he is studying at School of Electrical and Information Engineering, Jiangsu University of Technology, Changzhou, China and majored in communication engineering. His interests include signal processing and cognitive radio networks.

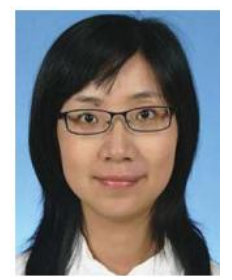

Lingjiao Pan received her BS in Information Engineering from Soochow University, China in 2004 and her M.S in information and communication engineering from Gwangju Institute of Science and Technology, Korea in 2007. Since 2008, she has been a member of engineering staff at Electronics and Telecommunications Research Institute(ETRI) Since 2012, she has been a lecturer at School of Electrical and Information Engineering, Jiangsu University of Technology, Changzhou, China, and her interests include video coding, image processing, 3-Dvideo, and depth video coding. 